\section{Simple material parameter estimation via terahertz time-domain spectroscopy}

W. Withayachumnankul, B. Ferguson, T. Rainsford, S.P. Mickan and D. Abbott

A simple and precise method based on fixed-point iteration is used to estimate dielectric parameters using terahertz time-domain spectroscopy (THz-TDS). The method converges and gives correct parameters when the sample thickness is greater than $200 \mu \mathrm{m}$ at a frequency of $0.1 \mathrm{THz}$ or $20 \mu \mathrm{m}$ at a frequency of $1.0 \mathrm{THz}$. The technique in validated using measured terahertz data, obtained by probing a sample of high-resistivity silicon.

Introduction: Terahertz or T-ray radiation lies in the $0.1-10 \mathrm{THz}$ frequency range, and has emerged to fill the gap between the upper limit of electronics and the lower limit of photonics. One of the most widely used terahertz applications is materials characterisation using terahertz time-domain spectroscopy (THz-TDS). With the coherent and ultra-wide bandwidth nature of a single-cycle terahertz pulse, we can extract amplitude and phase information from the signal at each individual frequency. This information leads to a specific material parameter, a complex refractive index, which becomes useful in describing and distinguishing many materials.

Background: Many parameter extraction methods for homogeneous materials based on a measurement of reference and probing terahertz signals have been proposed. The method introduced by Duvillaret et al. [1] models the error from the difference between estimated and measured data at each frequency. The error is then approximated by a paraboloid, and the complex refractive index at the apex of the paraboloid is found by a complicated numerical solution. Duvillaret et al. [2] and Dorney et al. [3] suggest a similar process for estimating the sample thickness. The process determines simultaneously a set of refractive indices at various guessed thicknesses, and uses the criterion of peak-to-peak or deepest total variation of the indices to select the correct thickness. This is applicable when the thickness of the sample is uncertain.

The method in this Letter is derived from a regular fixed-point iteration method. Provided with the reference and probing terahertz signals and the sample thickness, the method gives a simple, rapid, and precise solution to the problem. The solution is mathematically convergent at moderate or higher sample thicknesses. However, for a sample, which has a thickness comparable to or thinner than the wavelength, the sensitivity of terahertz time-domain spectroscopy is usually inadequate and it is then possible to use a more sensitive system exploiting terahertz differential time-domain spectroscopy instead [4].

Transfer function of material: Given a terahertz signal transmitted through the sample at normal incidence, $E_{s}(t)$, and a reference signal travelling an identical path without the presence of the sample, $E_{\text {ref }}(t)$, a measured transfer function, obtained by deconvolving the probing spectrum with respect to the reference spectrum, is described by [1]:

$$
\begin{aligned}
H_{\text {meas }}(\omega)= & \frac{E_{s}(\omega)}{E_{\text {ref }}(\omega)}=\frac{4 \tilde{n}_{s} \tilde{n}_{a}}{\left(\tilde{n}_{s}+\tilde{n}_{a}\right)^{2}} \\
& \times \exp \left[-i\left(\tilde{n}_{s}-\tilde{n}_{a}\right) \frac{\omega L}{c}\right] \cdot \operatorname{FP}(\omega)
\end{aligned}
$$

where $L$ is the sample thickness, and $\tilde{n}_{s}$ and $\tilde{n}_{a}$ are the frequencydependent complex refractive indices of sample and air, respectively. The complex refractive index contains two components: a real refractive index $n$ and an absorption index $\kappa$, where $\tilde{n}=n-i \kappa$. In $(1), \operatorname{FP}(\omega)$ represents the Fabry-Pérot effect or the interference in the received signal from reflections within the material,

$$
\operatorname{FP}(\omega)=\frac{1}{1-\left(\tilde{n}_{s}-\tilde{n}_{a} / \tilde{n}_{s}+\tilde{n}_{a}\right)^{2} \cdot \exp \left[-2 i \tilde{n}_{s}(\omega L / c)\right]}
$$

Model for material parameter estimation: The reflections or $\mathrm{FP}(\omega)$ in the transfer function should ideally be eliminated prior to the parameter estimation process. We can simply take out the reflections, by windowing the probing signal, if they are well separated from the primary pulse in the spatial domain. A more complicated procedure is given by Duvillaret et al. [1]. Assuming that preprocessing has taken place to remove the reflections from the measured signal, the argument of the transfer function is rearranged to,

$$
\begin{aligned}
n_{s} & =g_{1}\left(n_{s}, \kappa_{s}\right) \\
& =\frac{c}{\omega L}\left\{\arg \left[\frac{4 \tilde{n}_{s} \tilde{n}_{a}}{\left(\tilde{n}_{s}+\tilde{n}_{a}\right)^{2}}\right]-\arg \left[H_{\text {meas }}(\omega)\right]\right\}+n_{a}
\end{aligned}
$$

assuming that the absorption index of air or $\kappa_{a}$ equals to zero.

Also, we can find $\kappa_{s}$ from the transfer function by taking the logarithm of it and rearranging,

$$
\kappa_{s}=g_{2}\left(n_{s}, \kappa_{s}\right)=\frac{c}{\omega L}\left\{\ln \left|\frac{4 \tilde{n}_{s} \tilde{n}_{\text {air }}}{\left(\tilde{n}_{s}+\tilde{n}_{a}\right)^{2}}\right|-\ln \left|H_{\text {meas }}(\omega)\right|\right\}
$$

Equations (3) and (4) are in the form suitable for a fixed-point iteration in two dimensions. Provided the initial value of $n_{s}$ and $\kappa_{s}$ for the complex refractive index, the functions $g_{1}$ and $g_{2}$ map those points to new points. Repeatedly substituting the new values of $n_{s}$ and $\kappa_{s}$ into both functions gives updated values. If the functions appear convergent, when there is a small change in $n_{s}$ and $\kappa_{s}$ over the succeeding iterations, $n_{s}$ and $\kappa_{s}$ are then called fixed points of the functions.

If initial values of $n_{s}$ and $\kappa_{s}$, or $n_{0}$ and $\kappa_{0}$, are selected to be close to the final values, the iteration times can be reduced to some extent. Therefore, from (3) and (4) when $\left(4 \tilde{n}_{s} \tilde{n}_{a}\right) /\left(\tilde{n}_{s}+\tilde{n}_{a}\right)^{2}=1, n_{0}$ and $\kappa_{0}$ are given by

$$
\begin{aligned}
n_{0} & =-\frac{c}{\omega L} \arg \left[H_{\text {meas }}(\omega)\right]+n_{a} \\
\kappa_{0} & =-\frac{c}{\omega L} \ln \left|H_{\text {meas }}(\omega)\right|
\end{aligned}
$$

Condition of convergence: Given that both fixed-point functions, $g_{1}$ and $g_{2}$, are first-order differentiable, two sufficient conditions to guarantee the convergence of the functions are

$$
\left|\frac{\partial g_{1}}{\partial n_{s}}\right|+\left|\frac{\partial g_{1}}{\partial \kappa_{s}}\right|<1, \quad \text { and } \quad\left|\frac{\partial g_{2}}{\partial n_{s}}\right|+\left|\frac{\partial g_{2}}{\partial \kappa_{s}}\right|<1
$$

along the search path, i.e. from the initial value to the fixed point.

Substituting the derivatives of (3) and (4) into (6) gives the following inequality,

$$
\begin{aligned}
& \left|\frac{\kappa_{s}}{n_{s}^{2}+\kappa_{s}^{2}}-\frac{2 \kappa_{s}}{\left(n_{s}+n_{a}\right)^{2}+\kappa_{s}^{2}}\right| \\
& \quad+\left|\frac{n_{s}}{n_{s}^{2}+\kappa_{s}^{2}}-\frac{2\left(n_{s}+n_{a}\right)}{\left(n_{s}+n_{a}\right)^{2}+\kappa_{s}^{2}}\right|<\frac{\omega L}{c}
\end{aligned}
$$

To validate this condition, all possible values of $n_{s}$ and $\kappa_{s}$ are taken into account. Normally, a practical value [1] of refractive index $n_{s}$ lies between 1 and 10, whereas a practical value of attenuation index $\kappa_{s}$ lies between 0 and 10 . From the analysis, the maximum value for the LHS of (7) occurs at $n_{s}=1$ and $\kappa_{s}=0.9234$. By putting this critical point into (7), the condition of convergence is $0.4024<\omega L / c$. This condition implies that, at $f=0.1 \mathrm{THz}$, the minimum applicable thickness $L$ is $200 \mu \mathrm{m}$ and at $f=1.0 \mathrm{THz}$ the minimum applicable thickness is $20 \mu \mathrm{m}$.
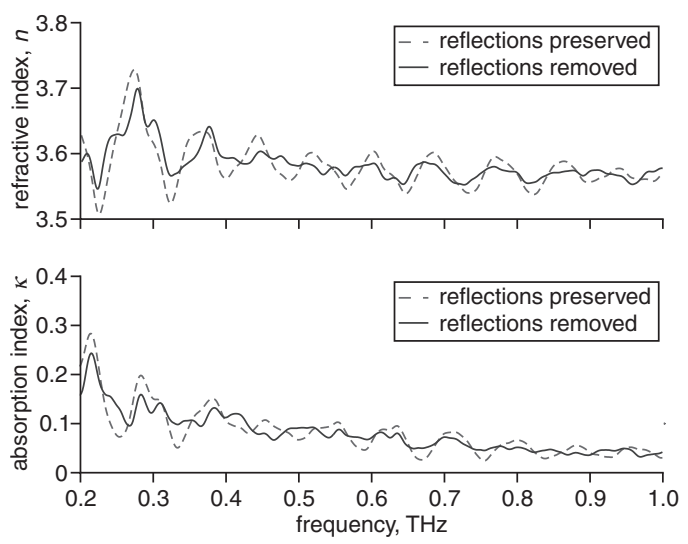

Fig. 1 Estimated indices of silicon wafer when reflections are preserved and removed by the process in [1] 
Result: The sample under test is a $500 \mu \mathrm{m}$-thick high-resistivity silicon wafer having the following characteristics: (i) polished on both sides, (ii) undoped, (iii) unbiased, (iv) $\langle 100\rangle$ crystal orientation, and (v) bulk silicon. The fixed point iteration is applied to the THzTDS data over an effective bandwidth with the number of iterations set to 5 at each frequency point. The extracted refractive index and absorption index, shown in Fig. 1, concur with results obtained in [5] in the 0.2 to $0.4 \mathrm{THz}$ range.

Conclusions: The simple parameter extraction method derived from the regular fixed-point iteration requires two terahertz signals, a sample signal and a reference signal, and the sample thickness to determine the complex refractive index. From the analysis, the method always produces a convergent solution when the thickness of sample is higher than $200 \mu \mathrm{m}$ at $0.1 \mathrm{THz}$ or $20 \mu \mathrm{m}$ at $1.0 \mathrm{THz}$, regardless of the refractive index value. This condition is applicable in most cases common to THz-TDS. Our method tested with a terahertz signal passing through a silicon wafer yields a promising result within a few iterations. Future work is required to improve further the removal of Fabry-Pérot effects.

(C) IEE 2005

Electronics Letters online no: 20051467

doi: 10.1049/el:20051467

W. Withayachumnankul, B. Ferguson, T. Rainsford, S.P. Mickan and D. Abbott (Centre for Biomedical Engineering and Department of Electrical \& Electronic Engineering, The University of Adelaide, SA 5005, Australia)

E-mail: dabbott@eleceng.adelaide.edu.au

\section{References}

1 Duvillaret, L., Garet, F., and Coutaz, J.L.: 'A reliable method for extraction of material parameters in terahertz time-domain spectroscopy', IEEE J. Sel. Top. Quantum Electron., 1996, 2, (3), pp. 739-746

2 Duvillaret, L., Garet, F., and Coutaz, J.L.: 'Highly precise determination of optical constants and sample thickness in terahertz time-domain spectroscopy', Appl. Opt., 1999, 38, (2), pp. 409-415

3 Dorney, T.D., Baraniuk, R.G., and Mittleman, D.M.: 'Material parameter estimation with terahertz time-domain spectroscopy', J. Opt. Soc. Am. A, 2001, 18, (7), pp. 1562-1571

4 Mickan, S.P., Lee, K.-S., Lu, T.-M., Munch, J., Abbott, D., and Zhang, X.-C.: 'Double modulated differential THz-TDS for thin film dielectric characterization', Microelectron. J., 2002, 33, (12), pp. $1033-1042$

5 Afsar, M.N., and Button, K.J.: 'Precise millimeter-wave measurements of complex refractive index, complex dielectric permittivity and loss tangent of $\mathrm{GaAs}, \mathrm{Si}, \mathrm{SiO}_{2}, \mathrm{Al}_{2} \mathrm{O}_{3}, \mathrm{BeO}$, Macor, and Glass', IEEE Trans. Microw. Theory Tech., 1983, MTT-31, (2), pp. 217-223 